\title{
Cognitive Dysfunction During Migraine Attacks
}

An Expert Interview with Raquel Gil-Gouveia

Hospital da Luz Headache Centre, Neurology Department, Hospital da Luz, Lisboa, Portugal

DOI: https://doi.org/10.17925/ENR.2019.14.1.12

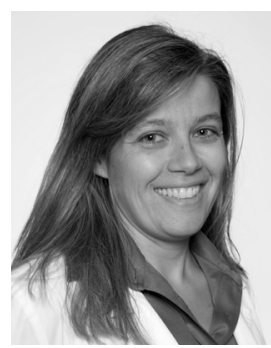

Raquel Gil-Gouveia MD, PhD

Raquel Gil-Gouveia MD, PhD is the Director of Neurology and of the Headache Centre in Hospital da Luz, Lisboa. She has been a researcher in headache since 1998, having worked in the clinical and pathophysiological aspects of cluster headache, headache associated with endovascular procedures and migraine. In migraine, she focused on the impact and disability of the migraine attack, in particular the cognitive aspects related to the brain changes occurring during attacks. She is part of the board of the Portuguese Headache Society, a European Academy of Neurology Scientist of the Panel on Headache and member of the Committee of the Lifting the Burden Global Campaign. She has participated in several international clinical trials in headache and other neurological disorders, is a frequent contributor to national and international meetings and advisory boards, and has published widely.

\section{Keywords}

Cognition, cognitive dysfunction, migraine

Disclosures: Raquel Gil-Gouveia has no conflicts of interest to declare in relation to this interview.

Acknowledgements: Medical writing

assistance was provided by Katrina Mountfort Freelance Writer for Touch Medical Media, and was supported by Touch Medical Media.

Review process: This is an expert interview and, as such, has not undergone the journal's standard peer review process but has been approved by the editorial board

Authorship: The named author meets the International Committee of Medical Journal Editors (ICMJE) criteria for authorship of this manuscript, take responsibility for the integrity of the work as a whole and have given final approval to the version to be published.

Received: 3 December 2018

Published Online: 30 April 2019

Citation: European Neurological Review. 2019:14(1):12-3

Corresponding Author: Raquel Gil-Gouveia, Centro de Cefaleias, Hospital da Luz Lisboa, Avenida Lusíada 100, 1500-650 Lisboa, Portugal. E: rgouveia@hospitaldaluz.pt

Support: No funding was received in

the publication of this article.
$\mathrm{M}$ igraine is the third most common disease in the world (behind dental caries and tension-type headache). It has an estimated global prevalence of $14.7 \%$, however, its importance tends to be overlooked in our society. ${ }^{1}$ Its impact relates to disability imposed by migraine attacks, during which over two-thirds of patients present some or total limitation of normal daily activities, such as work, study or daily chores. The two most important aspects that contribute to attack-related disability, are pain severity and cognitive symptoms. ${ }^{2}$ Patients with migraine, specifically those with active migraine and frequent attacks, are more likely to suffer from stroke, and to have silent white matter lesions. It has been suggested that migraine may be a progressive brain disorder and associated with an increased risk of cognitive impairment. ${ }^{2-4}$ Until recently, the relationship between migraine and cognitive deficits has only been investigated in cross-sectional and retrospective studies, which have yielded differing results. ${ }^{4,5}$ These conflicting reports might result from differences in the populations studied and heterogeneity of the neuropsychological tests used. 5

In an expert interview, Dr Raquel Gil-Gouveia discusses her recent randomised study, which prospectively assessed the association between migraine and cognitive function. ${ }^{6}$

\section{Q. How does migraine affect cognitive function?}

In one study, we aimed to evaluate if cognitive function was impaired during attacks; we included 39 patients, of which 24 completed the study. Patients underwent cognitive and behavioural tests during a spontaneously occuring, untreated migraine attack and a headache-free period. ${ }^{\circ}$ We found that, when patients are experiencing an attack of migraine without aura, they appear to undergo reversible brain dysfunction. ${ }^{6}$ We're not sure whether the migraine itself influences cognition, or whether how the brain processes the resulting pain is at the root of the problem.

To understand if these repeated attacks could contribute to later life cognitive dysfunction, we also studied the cognitive performance of population dwelling individuals over 50 years with persisting migraine and headaches. We found that there were no major differences in cognition in older patients with persisting attacks, nor was there any increased risk of cognitive decline.?

\section{Q. What aspects of cognitive performance are most affected?}

Our study found that word-reading speed, verbal learning, short-term verbal recall and delayed recall without semantic cues were the most affected functions when people were experiencing attacks. ${ }^{8}$ Other studies have found problems with attention, memory, language, visuospatial processing (ability to see and perceive the world) and executive functions (mental control and self-regulations). 


\section{Q. How does duration, intensity and frequency of migraines impact on cognitive function?}

The frequency and intensity of migraine and duration of attacks did not seem to influence this effect. We also found no associations with age, gender, literacy levels or anxiety. ${ }^{8}$

\section{Q. How do current migraine preventative medications affect cognitive function?}

some migraine prophylactic medications, such as topiramate, have been associated with cognitive dysfuntion. ${ }^{10}$ Some acute medication has been reported influence cognition in some patients, such as triptans. However, it is difficult to establish if these represent a medication side effect or the inability of acute medication to resolve attack-related cognitive symptoms. Our data highlight the importance of preventative medication to reduce the frequency of acute attacks in patients with migraine. It would be useful to conduct a study to look at whether cognition is improved when migraine is treated optimally. ${ }^{11}$

\section{Q. What changes in migraine management could help patients who suffer from cognitive impairment during migraine?}

These findings reinforce the fact that the management of migraine is more complex than only controlling the pain symptoms. It is important for patients who suffer cognitive complaints with migraine to realise that they may not maintain their usual intellectual performance during acute attacks, and to plan accordingly. Cognitive symptoms during migraines are extremely disabling and it is important to identify patients who suffer from cognitive symptoms at an early stage and adjust therapeutic options. It is reasonable to evaluate cognitive symptomatology routinely but if cognitive dysfunction is identified while in a headache-free status, it is essential to rule out other potential causes of impaired cognition, such as thyroid disorders or severe anxiety or mood disorders. We still need further data to form firm conclusions. We are currently performing functional cerebral imaging exams during migraine attacks to evaluate the activity of the brain. $\square$

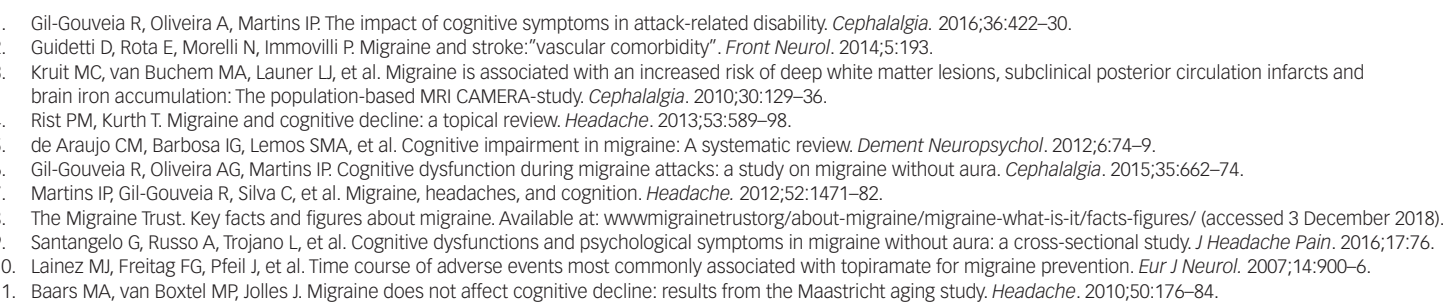

